\title{
High voltage calibration method for the CMS RPC detector
}

To cite this article: R. Reyes-Almanza et al 2019 JINST 14 C09046

View the article online for updates and enhancements.

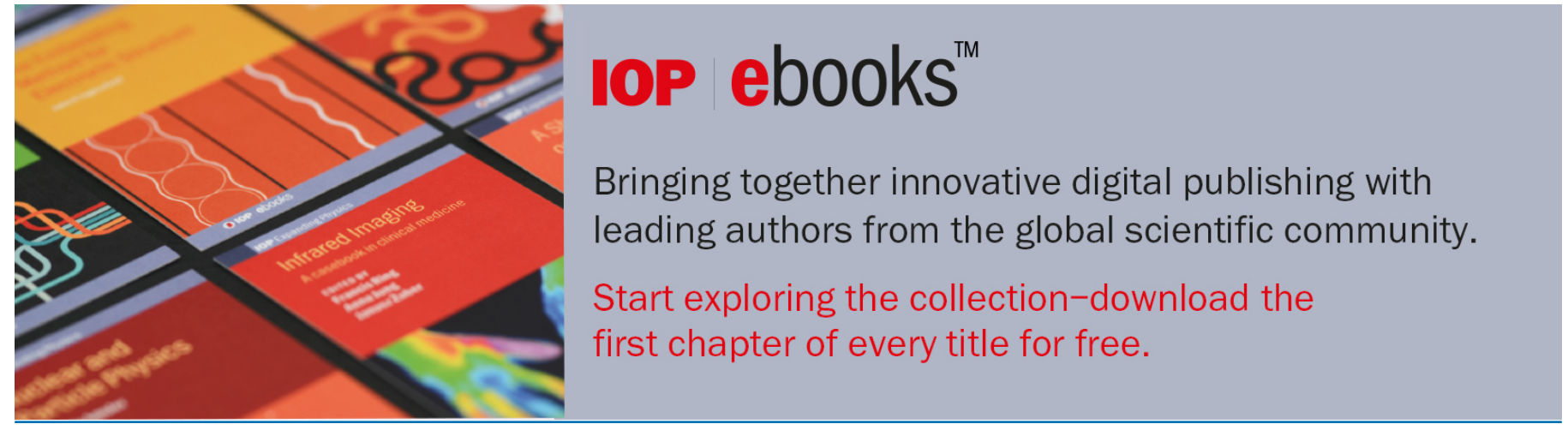

This content was downloaded from IP address 157.193 .149 .49 on $24 / 09 / 2020$ at 13:18 


\section{High voltage calibration method for the CMS RPC detector}

R. Reyes-Almanza, ${ }^{x, 1}$ A. Dimitrov,,${ }^{d, 1}$ A. Fagot, ${ }^{a}$ M. Gul, ${ }^{a}$ C. Roskas, ${ }^{a}$ M. Tytgat, ${ }^{a}$ N. Zaganidis, ${ }^{a}$ S. Fonseca De Souza, ${ }^{b}$ A. Santoro, ${ }^{b}$ F. Torres Da Silva De Araujo, ${ }^{b}$ A. Aleksandrov, ${ }^{c}$ R. Hadjiiska, ${ }^{c}$ P. laydjiev, ${ }^{c}$ M. Rodozov, ${ }^{c}$ M. Shopova, ${ }^{c}$ G. Sultanov, ${ }^{c}$ L. Litov, ${ }^{d}$ B. Pavlov, ${ }^{d}$ P. Petkov, ${ }^{d}$ A. Petrov, ${ }^{d}$ S.J. Qian, ${ }^{e}$ D. Han, W. $\mathbf{Y i}^{f}$ C. Avila, ${ }^{g}$ A. Cabrera, ${ }^{g}$ C. Carrillo, ${ }^{g}$ M. Segura, ${ }^{g}$ S. Aly, ${ }^{h}$ Y. Assran, ${ }^{h}$ A. Mahrous, ${ }^{h}$ A. Mohamed, ${ }^{h}$ C. Combaret ${ }^{i}$ M. Gouzevitch, ${ }^{i}$ G. Grenier, ${ }^{i}$ F. Lagarde,${ }^{i}$ I.B. Laktineh, ${ }^{i}$ H. Mathez ${ }^{i}$ L. Mirabito, ${ }^{i}$ K. Shchablo, ${ }^{i}$ I. Bagaturia,${ }^{j}$ D. Lomidze,${ }^{j}$ I. Lomidze,${ }^{j}$ L.M. Pant,${ }^{k}$ V. Bhatnagar, ${ }^{l}$ R. Gupta, ${ }^{l}$ R. Kumari, ${ }^{l}$ M. Lohan, ${ }^{l}$ J.B.Singh,${ }^{l}$ V. Amoozegar, ${ }^{m}$ B. Boghrati, ${ }^{m, n}$ H. Ghasemy, ${ }^{m}$ S. Malmir, ${ }^{m}$ M. Mohammadi Najafabadi, ${ }^{m}$ M. Abbrescia, ${ }^{o}$ A. Gelmi,${ }^{o}$ G. laselli, ${ }^{o}$ S. Lezki, ${ }^{o}$ G. Pugliese,${ }^{o}$ L. Benussi, ${ }^{p}$ S. Bianco, ${ }^{p}$ D.Piccolo, ${ }^{p}$ F. Primavera, ${ }^{p}$ S. Buontempo, ${ }^{q}$ A. Crescenzo, ${ }^{q}$ G. Galati, ${ }^{q}$ F. Fienga,${ }^{q}$ I. Orso,${ }^{q}$ L. Lista, ${ }^{q}$ S. Meola, ${ }^{q}$ P. Paolucci, ${ }^{q}$ E. Voevodina, ${ }^{q}$ A. Braghieri, ${ }^{r}$ P. Montagna,${ }^{r}$ M. Ressegotti, ${ }^{r}$ C. Riccardi, ${ }^{r}$ P. Salvini, ${ }^{r}$ P. Vitulo, ${ }^{r}$ S.W. Cho, ${ }^{s}$ S.Y. Choi, ${ }^{s}$ B. Hong, ${ }^{s}$ K.S. Lee, ${ }^{s}$ J.H. Lim, ${ }^{s}$ S.K. Park, ${ }^{s}$ J. Goh, ${ }^{t, u}$ T.J. Kim, ${ }^{t}$ S. Carrillo Moreno, ${ }^{v}$ O. Miguel Colin, ${ }^{v}$ F. Vazquez Valencia, ${ }^{v}$ S. Carpinteyro Bernardino, ${ }^{w}$ J. Eysermans, ${ }^{w}$ I. Pedraza, ${ }^{w}$ C. Uribe Estrada, ${ }^{w}$ M.C. Duran-Osuna, ${ }^{x}$ M. Ramirez-Garcia, ${ }^{x}$ G. Ramirez-Sanchez, ${ }^{x}$ A. Sanchez-Hernandez, ${ }^{x}$ R.I. Rabadan-Trejo, ${ }^{x}$ H. Castilla-Valdez, ${ }^{x}$ A. Radi, ${ }^{y}$ H. Hoorani, ${ }^{z}$ S. Muhammad, ${ }^{z}$ M.A. Shah ${ }^{z}$ and I. Crotty ${ }^{a a}$ on behalf of CMS muon group

${ }^{a}$ Ghent University, Dept. of Physics and Astronomy, Proeftuinstraat 86, B-9000 Ghent, Belgium

${ }^{b}$ Dep. de Fisica Nuclear e Altas Energias, Instituto de Fisica, Universidade do Estado do Rio de Janeiro, Rua Sao Francisco Xavier, 524, BR - Rio de Janeiro 20559-900, RJ, Brazil

${ }^{c}$ Bulgarian Academy of Sciences, Inst. for Nucl. Res. and Nucl. Energy, Tzarigradsko shaussee Boulevard 72, BG-1784 Sofia, Bulgaria

${ }^{d}$ Faculty of Physics, University of Sofia,5 James Bourchier Boulevard, BG-1164 Sofia, Bulgaria

e School of Physics, Peking University, Beijing 100871, China

${ }^{f}$ Tsinghua University, Shuangqing Rd, Haidian Qu, Beijing, China

${ }^{g}$ Universidad de Los Andes, Apartado Aereo 4976, Carrera 1E, no. 18A 10, CO-Bogota, Colombia

${ }^{h}$ Egyptian Network for High Energy Physics, Academy of Scientific Research and Technology, 101 Kasr El-Einy St. Cairo Egypt

\footnotetext{
${ }^{1}$ Corresponding authors.
} 
${ }^{i}$ Universite de Lyon, Universite Claude Bernard Lyon 1, CNRS-IN2P3, Institut de Physique Nucleaire de Lyon, Villeurbanne, France

${ }^{j}$ Georgian Technical University, 77 Kostava Str., Tbilisi 0175, Georgia

${ }^{k}$ Nuclear Physics Division Bhabha Atomic Research Centre Mumbai 400 085, India

${ }^{l}$ Department of Physics, Panjab University, Chandigarh Mandir 160 014, India

${ }^{m}$ School of Particles and Accelerators, Institute for Research in Fundamental Sciences (IPM), Tehran, Iran

${ }^{n}$ School of Engineering, Damghan University, Damghan, Iran

o INFN, Sezione di Bari, Via Orabona 4, IT-70126 Bari, Italy

${ }^{p}$ INFN, Laboratori Nazionali di Frascati (LNF), Via Enrico Fermi 40, IT-00044 Frascati, Italy

q INFN, Sezione di Napoli, Complesso Univ. Monte S. Angelo, Via Cintia, IT-80126 Napoli, Italy

${ }^{r}$ INFN, Sezione di Pavia, Via Bassi 6, IT-Pavia, Italy

${ }^{s}$ Korea University, Department of Physics, 145 Anam-ro, Seongbuk-gu, Seoul 02841, Republic of Korea

${ }^{t}$ Hanyang University, 222 Wangsimni-ro, Sageun-dong, Seongdong-gu, Seoul, Republic of Korea

${ }^{u}$ Kyunghee University, 26 Kyungheedae-ro, Hoegi-dong, Dongdaemun-gu, Seoul, Republic of Korea

${ }^{v}$ Universidad Iberoamericana, Mexico City, Mexico

${ }^{w}$ Benemerita Universidad Autonoma de Puebla, Puebla, Mexico

${ }^{x}$ Cinvestav, Av. Instituto Politécnico Nacional No. 2508, Colonia San Pedro Zacatenco, CP 07360, Ciudad de Mexico D.F., Mexico

${ }^{y}$ Sultan Qaboos University, Al Khoudh,Muscat 123, Oman

${ }^{z}$ National Centre for Physics, Quaid-i-Azam University, Islamabad, Pakistan

${ }^{a a}$ Dept. of Physics, Wisconsin University, Madison, WI 53706, United States

E-mail: rogelio.reyes.almanza@cern.ch

AbstRact: The Resistive Plate Chambers (RPC) are used for muon triggers in the CMS experiment. To calibrate the high voltage working-points (WP) and identify degraded detectors due to radiation or chemical damage, a high voltage scan has been performed using 2017 data from pp collisions at a center-of-mass energy of $13 \mathrm{TeV}$. In this paper, we present the calibration method and the latest results obtained for the 2017 data. A comparison with all scans taken since 2011 is considered to investigate the stability of the detector performance in time.

Keywords: Detector alignment and calibration methods (lasers, sources, particle-beams); Performance of High Energy Physics Detectors; Resistive-plate chambers 


\section{Contents}

1 Introduction 1

2 Calibration method 1

3 Results 2

3.1 The WP definition 2

3.2 The stability of the detector 3

4 Summary and conclusions $\quad 4$

\section{Introduction}

The Compact Muon Solenoid (CMS) [1] whose central feature is a superconducting solenoid magnet providing a magnetic field of $3.8 \mathrm{~T}$ has been used to study a wide range of physics phenomena generated in hadron collisions at $\sqrt{s}=13 \mathrm{TeV}$. In the CMS, muons are detected in gas-ionization chambers within the pseudorapidity range $|\eta|<2.4$, they are distributed in detection planes and using three technologies: drift tubes (DT), cathode strip chambers (CSC), and resistive plate chambers (RPCs). The present RPC system covering $|\eta|<1.9$ consists of a total of 1056 double-gap chamber modules, and is divided in $\eta$ partitions so-called "rolls". The system is designed as muon trigger detector and contributes to all muon track finders, a detailed description is discussed in reference [2].

The calibrations of high voltage (HV) for the RPC detector are performed by a HV scan measurement once per year using a special calibration run or sequence of runs. Establishing the correct operational working points in HV for the individual RPC detector is of primary importance, to ensure a stable detector performance, to provide the optimal efficiency, and to keep the cluster size required for the CMS triggers [2]. In addition, the periodic HV scan measurements enable us to study the long-term stability of the detectors and to identify the potential degradation due to "aging". In 2017 two HV scans were performed with different instantaneous luminosities in pp collisions at $\sqrt{s}=13 \mathrm{TeV}$ and different concentrations of the operational gas mixture. In this report, the results of the first HV scan performed in 2017 are presented and compared with the previous results obtained from 2011 to 2017.

\section{Calibration method}

The data are collected in a particular configuration of the detector, varying the high voltage values within a HV range from 8800 to $9800 \mathrm{~V}$. Events of interest are selected using the DT and CSC trigger and reconstructed using the CMS standard muon reconstruction [2]. A linear extrapolation of track segment in DT and CSC chambers was performed toward the closest RPC strip plane, and then matched to any RPC cluster in a range of 8 strips around the extrapolated impact point. This method provides a measure for the efficiency, which is defined as the ratio of number of 


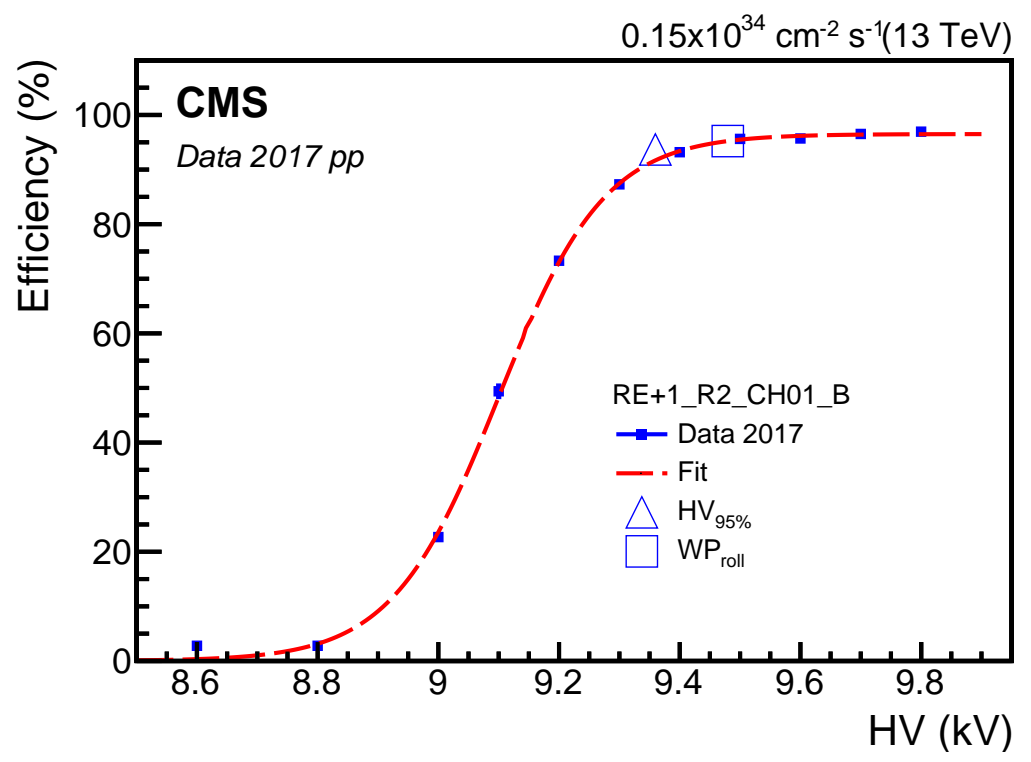

Figure 1. Efficiency as a function of HV measured for an endcap RPC. The red dashed curve represents the performed fit using a sigmoid function.

tracks detected in the RPC divided by the number of extrapolated tracks identified in CSC and DT detectors. The efficiency is measured for every HV point and the resulting distribution is fitted chamber by chamber using a sigmoid function defined as

$$
\varepsilon=\frac{\varepsilon_{\max }}{1+\exp \left(-\lambda\left(\mathrm{HV}-\mathrm{HV}_{50 \%}\right)\right)},
$$

where $\lambda$ characterize the slope of the sigmoid, $\varepsilon_{\max }$ represents the plateau of the distribution, and $\mathrm{HV}_{50 \%}$ is the value of the voltage at $50 \%$ of the maximum efficiency. The HV where the efficiency reaches $95 \%\left(\mathrm{HV}_{95 \%}\right)$ of the maximum efficiency $\left(\varepsilon_{\max }\right)$ is obtained by applying the sigmoid functional fit to the efficiency data and by interpolating the function. The working points (WPs) for the RPCs in the barrel and endcap regions are defined as $\mathrm{HV}_{95 \%}+100 \mathrm{~V}$ and $\mathrm{HV}_{95 \%}+120 \mathrm{~V}$, respectively. Since the counting rate is different in both regions, the definition of the WPs allows to measure a similar global efficiency throughout detector. An example of the sigmoid functional fit and the efficiencies measured for an endcap RPC is shown in figure 1. The distributions of WPs and the efficiencies at the WPs for the barrel, the endcap detectors at RE1, 2 and 3 station and at RE4 station are shown in figure 2.

The RPCs which cannot provide the proper data required for the present study are excluded. They are the chambers whose efficiency data obtained from the tracker detectors are missing. The RPCs operated in a single-gap mode because of gas leak or HV problem are also excluded from the analysis.

\section{Results}

\subsection{The WP definition}

A total 480 and $293 \mathrm{HV}$ channels in barrel and endcap regions, respectively, supply between 2 barrel and 6 endcap rolls. The working point in the channel $\left(\mathrm{WP}_{\mathrm{CH}}\right)$ is computed on the basis of the values 

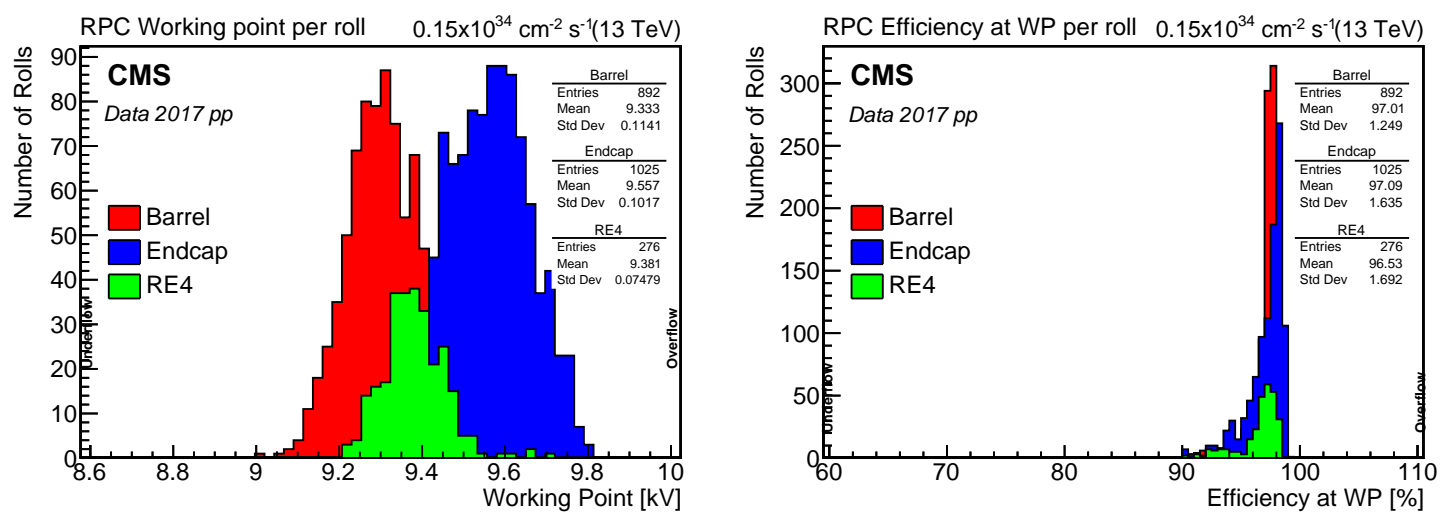

Figure 2. The WPs and efficiency at WPs distribution computed after fit results. The red sample represents the distributions for the barrel rolls. The blue sample represents the distribution for endcap rolls at RE1, RE2, and RE3 stations, and the green sample presents the lately installed endcap RE4. The RPCs which cannot provide proper data are excluded.
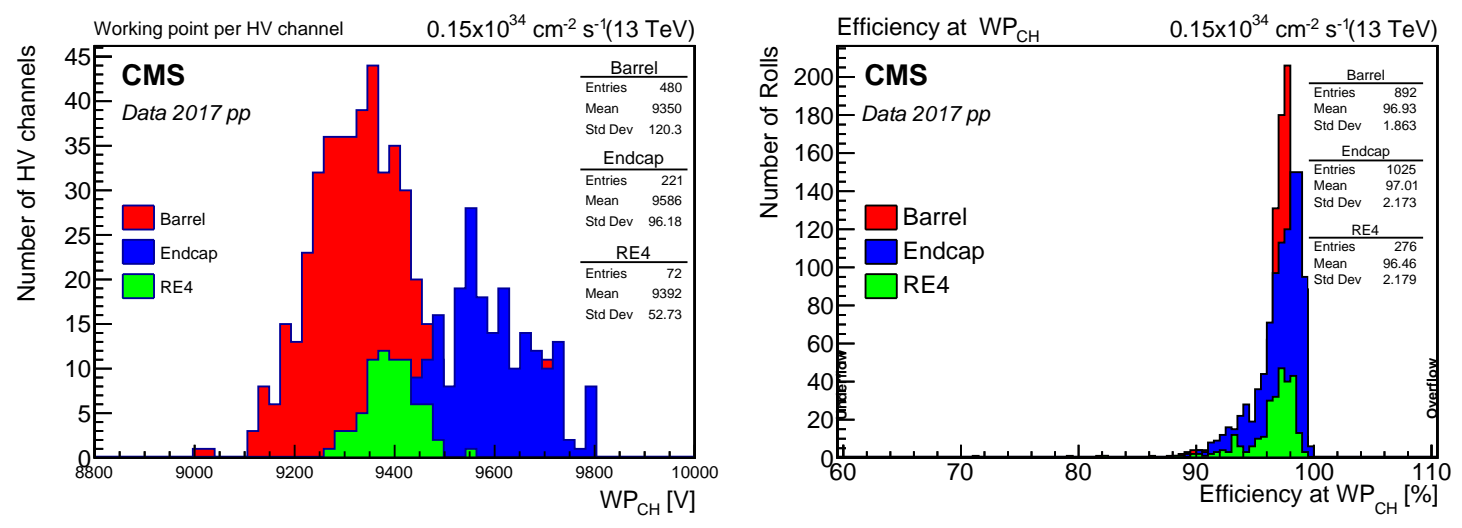

Figure 3. The final results for WP in HV channels to be applied (left) and the distribution of the efficiency at $\mathrm{WP}_{\mathrm{CH}}$ (right). The red sample represents the distributions for the barrel rolls. The blue sample represents the distribution for endcap rolls at RE1, RE2, and RE3 stations, and the green sample presents the lately installed endcap RE4. The RPCs which cannot provide proper data are excluded.

of each WP per roll ( $\left.\mathrm{WP}_{\text {roll }}\right)$ in a single $\mathrm{HV}$ channel, by taking into account the spread between the maximum and the minimum of the $\mathrm{WPs}_{\text {roll }}$ values. The $\mathrm{WP}_{\mathrm{CH}}$ is defined by the following:

$$
\mathrm{WP}_{\mathrm{CH}}=\left\{\begin{array}{lll}
\left\langle\mathrm{WP}_{\text {roll }}\right\rangle & \text { if } & \mathrm{WP}_{\text {roll }}^{\mathrm{Max}}-\mathrm{WP}_{\text {roll }}^{\mathrm{Min}} \leq 100 \mathrm{~V} \\
\mathrm{WP}_{\text {roll }}^{\mathrm{Min}}+100 \mathrm{~V} & \text { if } & \mathrm{WP}_{\text {roll }}^{\mathrm{Max}}-\mathrm{WP}_{\text {roll }}^{\mathrm{Min}}>100 \mathrm{~V}
\end{array}\right.
$$

here $\mathrm{WP}_{\text {roll }}^{\mathrm{Max}}$ and $\mathrm{WP}_{\text {roll }}^{\mathrm{Min}}$ represent the maximum and minimum of $\mathrm{WP}_{\text {roll }}$, respectively, and $<$ $\mathrm{WP}_{\text {roll }}>$ represent the average of the $\mathrm{WPs}_{\text {roll }}$ values for each $\mathrm{HV}$ channel. The $\mathrm{WP}_{\mathrm{CH}}$ has been determined for 400 and $271 \mathrm{HV}$ channels in the barrel and endcap region, respectively, the results for $\mathrm{WP}_{\mathrm{CH}}$ and the efficiencies at those values are shown in figure 3.

\subsection{The stability of the detector}

For this study 6 HV scans taken since 2011 are considered. From the standard 2011 to 2017 HV scans are usually taken during the first calibration runs with collisions at the beginning and the 

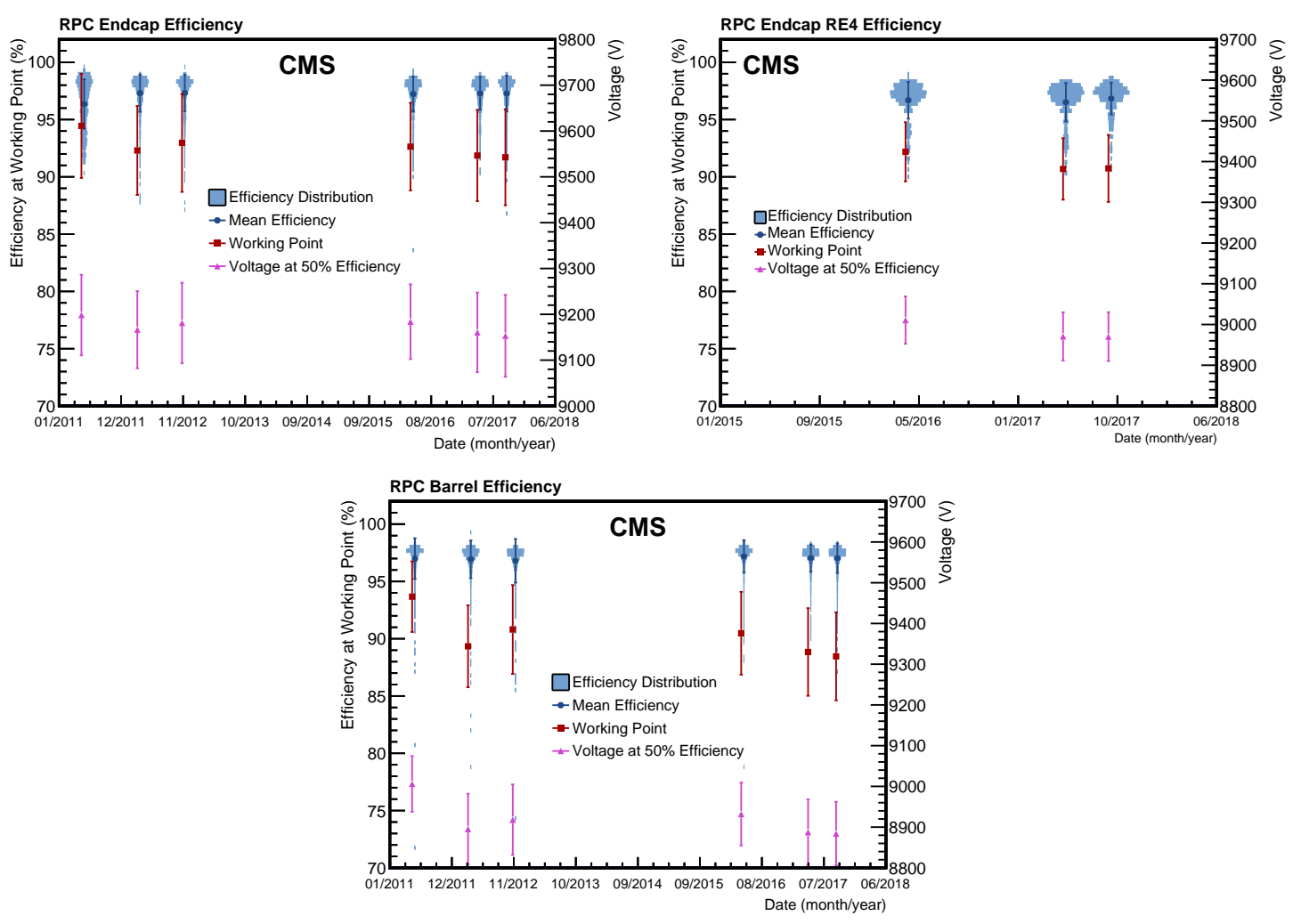

Figure 4. Results from HV scans per year, used to explore the WP evolution, the efficiency at WP evolution and the $\mathrm{HV}_{50 \%}$ for endcap (top plots) and barrel (bottom plot) rolls. The efficiency at WP distributions have been presented with a light blue color. With a blue, full circle is presented the mean efficiency at WP for each of the HV scans. By red, full squares is represented the mean of the working point distribution for each HV scan with their standard deviations. In magenta, full triangles represent the mean of the $\mathrm{HV}_{50 \%}$ distribution for each HV scan with their standard deviations.

middle of each operational year, and to consider different instantaneous luminosity conditions. Due to the long shutdown one maintenance period in the Large Hadron Collider, the RPC detector was OFF, therefore no HV scans were performed in 2013 and 2014. The 2015 scan was omitted in order to avoid introducing unknown bias to the study due to the missing magnetic field. The same selection criteria is used to compute the efficiency and also the same rolls are considered. The results are shown in figure 4. As it can be seen in figure 4, a shift of about 40 and $20 \mathrm{~V}$ to lower values for the barrel and endcap rolls with respect to last year scan. This behavior is due to a higher concentration of isobutane (5.2\%) in the RPC gas mixture on the last year, a high efficiency is kept despite this change.

\section{Summary and conclusions}

In 2017 two datasets have been taken with different luminosity conditions, in pp collisions at $\sqrt{s}=13 \mathrm{TeV}$. The HV scan calibration method is an optimal method to obtain the best WP values to the HV channel, and it has been used with profit in more than $84 \%$ of the chambers in the barrel, 
and $94 \%$ in the endcap. The presented results in figure 3 show the expected efficiency around $97 \%$ in the detector when the computed $\mathrm{WP}_{\mathrm{CH}}$ is applied.

A shift of 40 and $20 \mathrm{~V}$ to lower values, in the barrel and endcap, has been observed with respect to last year scan, because of a higher concentration of isobutane (5.2\%) in the RPC gas mixture. The changes in the mixture does not the affect the efficiency.

\section{References}

[1] CMS collaboration, The CMS Experiment at the CERN LHC, 2008 JINST 3 S08004.

[2] CMS collaboration, Performance of the CMS muon detector and muon reconstruction with proton-proton collisions at $\sqrt{s}=13 \mathrm{TeV}, 2018$ JINST 13 P06015 [arXiv: 1804. 04528]. 